\title{
A CHANGE OF SCALE FORMULA FOR WIENER INTEGRALS ON ABSTRACT WIENER SPACES
}

\author{
IL YOO \\ Department of Mathematics \\ Yonsei University \\ Kangwondo, 222-701, Korea \\ DAVID SKOUG \\ Department of Mathematics \& Statistics \\ University of Nebraska \\ Lincoln, NE 68588-0323 \\ (Received October 15, 1992)
}

\begin{abstract}
In this paper we obtain a change of scale formula for Wiener integrals on abstract Wiener spaces. This formula is shown to hold for many classes of functions of interest in Feynman integration theory and quantum mechanics.
\end{abstract}

KEY WORDS AND PHRASES. Abstract Wiener space, Wiener integral, Feynman integral.

1991 AMS SUBJECT CLASSIFICATION CODE. 28C20

\section{INTRODUCTION.}

It has long been known that Wiener measure and Wiener méasurability behave badly under the change of scale transformation [1] and under translations [2]. For many problems, this pathology causes no special difficulties. However in the theory of the analytic Wiener and analytic Feynman integrals one considers functionals of the form $F(\lambda x)$ where $\lambda$ varies over the positive reals and $\mathrm{x}$ varies over Wiener space. Johnson and Skoug [3] showed that scale-invariant measurability was the appropriate setting for the analytic Wiener and Feynman integration theories. This concept was extended to Yeh-Wiener space by Chang [4] and to abstract Wiener space by Chung [5].

Cameron and Storvick [6], for a rather large class of functionals, expressed the analytic Wiener integral as a limit of Wiener integrals. In doing so they discovered a rather nice change of scale formula for Wiener integrals [7]. In [8], Yoo extended these results to Yeh-Wiener space. The purpose of this paper is to obtain a change of scale formula for Wiener integrals on an abstract Wiener space. Results in $[6,7,8]$ are then corollaries of our results. Finally, we note that the Wiener integral of many classes of 
functionals of interest in Feynman integration theory and quantum mechanics [9-18] satisfy this change of scale formula.

\section{DEFINITIONS AND PRELIMINARIES.}

Let $\mathrm{H}$ be a real separable infinite dimensional Hilbert space with inner product $\langle\cdot, \cdot\rangle$ and norm $\|\cdot\|$. Let $\|\cdot \cdot\|$ be a measurable norm on $H$ with respect to the Gaussian cylinder set measure $\sigma$ on $\mathrm{H}$. Let $\mathrm{B}$ denote the completion of $\mathrm{H}$ with respect to $\|\cdot \mid\| \cdot$. Let $\mathrm{i}$ denote the natural injection from $H$ into $B$. The adjoint operator $i^{*}$ of $i$ is one-to-one and maps $\mathrm{B}^{*}$ continuously onto a dense subset of $\mathrm{H}^{*}$. By identifying $\mathrm{H}$ with $\mathrm{H}^{*}$ and $\mathrm{B}^{*}$ with $\mathrm{i}^{*} \mathrm{~B}^{*}$, we have a triple $B^{*} C H^{*} \equiv H C B$ and $\langle h, x\rangle=(h, x)$ for all $h$ in $H$ and $x$ in $B^{*}$, where $(\cdot, \cdot)$ denotes the natural dual pair between $B$ and $B^{*}$. By a well known result of Gross [19], $\sigma \circ \mathrm{i}^{-1}$ has a unique countably additive extension $\nu$ to the Borel $\sigma$-algebra $\mathscr{B}(\mathrm{B})$ of $\mathrm{B}$. The triple $(\mathrm{H}, \mathrm{B}, \nu)$ is called an abstract Wiener space and the Hilbert space $\mathrm{H}$ is called the generator of $(H, B, \nu)$. For more detail see $[19,20]$.

Let $\left\{e_{j}\right\}_{j=1}^{\infty}$ denote a complete orthonormal (CON) system in $H$ with the $e_{j}$ 's in $B^{*}$. For each $h \in H$ and $x \in B$, define a stochastic inner product $(\cdot, \cdot)^{\sim}$ between $H$ and $B$ as follows:

$$
(\mathrm{h}, \mathrm{x})^{\sim}= \begin{cases}\lim _{\mathrm{n} \rightarrow \infty} \sum_{k=1}^{\mathrm{n}}\left\langle\mathrm{h}, \mathrm{e}_{\mathbf{k}}\right\rangle\left(\mathrm{x}, \mathrm{e}_{\mathbf{k}}\right), & \text { if the limit exists } \\ 0 & , \text { otherwise }\end{cases}
$$

It is well known that for every $h \in H,(h, x)^{\sim}$ exists for $\nu$-a.e.x $\in B$ and is a Borel measurable function of $B$ having a Gaussian distribution with mean zero and variance $\|\mathrm{h}\|^{2}$. Furthermore, it is easy to show that $(h, x)^{\sim}=(h, x) \nu$-a.e. on $B$ if $h \in B^{*}$. Note that if both $\mathrm{h}$ and $\mathrm{x}$ are in $\mathrm{H}$, then $(\mathrm{h}, \mathrm{x})^{\sim}=\langle\mathrm{h}, \mathrm{x}\rangle$.

Let $\mathrm{M}(\mathrm{H})$ denote the class of $\mathrm{C}$-valued countably additive measures defined on $\mathscr{B}(\mathrm{H})$, the Borel class of $\mathrm{H}$. $\mathrm{M}(\mathrm{H})$ is a Banach algebra under the total variation norm and with convolution as multiplication.

Given two $C$-valued functions $F$ and $G$ on $B$, we say that $F=G$ s-a.e. if for each $\alpha>0, F(\alpha x)=G(\alpha x)$ for $\nu$-a.e. $x \in B$. For a function $F$ on $B$, let $[F]$ denote the equivalence class of functionals which are equal to $\mathrm{F}$ s-a.e..

The Fresnel class $\mathscr{F}(\mathrm{B})$ of functions on $\mathrm{B}$ is defined as the space of all functions $\mathrm{F}$ on B of the form

$$
\mathbf{F}(\mathbf{x})=\int_{\mathbf{H}} \exp \left\{\mathrm{i}(\mathrm{h}, \mathbf{x})^{\sim}\right\} \mathrm{d} \mu(\mathrm{h})
$$

for some $\mu \in \mathrm{M}(\mathrm{H})$. More precisely, since we identify functions which coincide s-a.e. on $\mathrm{B}$, $\mathscr{F}(\mathrm{B})$ is the space of all $\mathrm{s}$-equivalence classes of functions of the form (2.2). It is well-known $[18,19]$ that $\mathscr{F}(\mathrm{B})$ is a Banach algebra and the mapping $\mu \rightarrow \mathrm{F}$ is a Banach algebra isomorphism where $\mu$ and $\mathrm{F}$ are related by (2.2).

DEFINITION 1. Let $\mathrm{F}$ be a $\mathrm{C}$-valued measurable function on $\mathrm{B}$ such that the integral

$$
\mathrm{J}_{\mathrm{F}}(\lambda)=\int_{\mathbf{B}} \mathrm{F}\left(\lambda^{\frac{-1}{2}} \mathbf{x}\right) \mathrm{d} \nu(\mathbf{x})
$$

exists for all real $\lambda>0$. If there exists an analytic function $\mathrm{J}_{\mathrm{F}}^{*}(\lambda)$ on $\mathbb{C}^{+}=\{\lambda \in \mathbb{C}: \operatorname{Re} \lambda>0\}$ such that $\mathrm{J}_{\mathrm{F}}^{*}(\lambda)=\mathrm{J}_{\mathrm{F}}(\lambda)$ for all real $\lambda>0$, then we define $\mathrm{J}_{\mathrm{F}}^{*}(\lambda)$ to be the analytic Wiener integral of $\mathrm{F}$ over $\mathrm{B}$ with parameter $\lambda$, and for $\lambda \in \mathbb{C}^{+}$, we write 


$$
\mathrm{I}_{\mathrm{a}}^{\lambda}(\mathrm{F})=\mathrm{J}_{\mathrm{F}}^{*}(\lambda)
$$

For real $\mathrm{q} \neq 0$, if the following limit exists, we call it the analytic Feynman integral of $\mathrm{F}$ over $B$ with parameter $q$ and we write

$$
\mathrm{I}_{\mathrm{a}}^{\mathrm{q}}(\mathrm{F})=\lim _{\lambda \rightarrow \mathrm{iq}} \mathrm{I}_{\mathrm{a}}^{\lambda}(\mathrm{F})
$$

where $\lambda$ approaches - iq through values in $\mathrm{C}^{+}$.

THEOREM $1([14,15])$. Let $F \in \mathscr{F}(\mathrm{B})$ be given by (2.2). Then the analytic Feynman integral of $F$ over $B$ exists for all real $q \neq 0$ and

$$
I_{a}^{q}(F)=\int_{H} \exp \left\{-\frac{i}{2 q}\|h\|^{2}\right\} d \mu(h) .
$$

In addition for each $\lambda \in \mathbf{C}^{+}$,

$$
\mathrm{I}_{\mathbf{a}}^{\lambda}(\mathrm{F})=\int_{\mathrm{H}} \exp \left\{-\frac{1}{2 \lambda}\|\mathrm{h}\|^{2}\right\} \mathrm{d} \mu(\mathrm{h}) .
$$

3. A CHANGE OF SCALE FORMULA FOR WIENER INTEGRALS ON ABSTRACT WIENER SPACES.

We begin this section with a key lemma for Wiener integrals on an abstract Wiener space $(\mathrm{H}, \mathrm{B}, \nu)$.

LEMMA 1. Let $\lambda \in \mathrm{C}^{+}$, let $\left\{\mathrm{e}_{1}, \cdots, \mathrm{e}_{\mathrm{n}}\right\}$ be an orthonormal set in $\mathrm{H}$ and let $\mathrm{h} \in \mathrm{H}$. Then

$$
\begin{aligned}
\int_{B} \exp \left\{\left[\frac{1-\lambda}{2}\right] \sum_{\mathbf{k}=1}^{\mathrm{n}}\left[\left(\mathrm{e}_{\mathbf{k}}, \mathbf{x}\right)^{\sim}\right]^{2}+\mathrm{i}(\mathrm{h}, \mathrm{x})^{\sim}\right\} \mathrm{d} \nu(\mathrm{x}) \\
=\lambda^{\frac{-\mathrm{n}}{2}} \exp \left\{\left[\frac{\lambda-1}{2 \lambda}\right] \sum_{\mathbf{k}=1}^{\mathrm{n}}\left[\left\langle\mathrm{e}_{\mathrm{k}}, \mathrm{h}\right\rangle\right]^{2}-\frac{\|\mathrm{h}\|^{2}}{2}\right\} .
\end{aligned}
$$

PROOF. Using the Gram-Schmidt process we obtain $e_{n+1} \in H$ such that $\left\{e_{1}, \cdots, e_{n+1}\right\}$ forms an orthonormal set in $H$ and $h=\underset{k=1}{n+1} c_{k} e_{k}$ where

$$
c_{k}= \begin{cases}\left\langle e_{k}, h\right\rangle & , k=1, \cdots, n \\ \left(\|h\|^{2}-\sum_{k=1}^{n}\left[\left\langle e_{k}, h\right\rangle\right]^{2}\right)^{\frac{1}{2}}, \mathbf{k}=n+1 .\end{cases}
$$

Also since $\left(e_{1}, x\right)^{\sim}, \cdots,\left(e_{n+1}, x\right)^{\sim}$ are independent Gaussian random variables and since

$$
\int_{\mathbb{R}} \exp \left\{-a y^{2}+i b y\right\} d y=\left(\frac{\pi}{a}\right)^{\frac{1}{2}} \exp \left\{-\frac{b^{2}}{4 a}\right\}, a \in \mathbb{C}^{+}
$$

we obtain that

$$
\begin{aligned}
& \int_{\mathbf{B}} \exp \left\{\left[\frac{1-\lambda}{2}\right] \sum_{\mathbf{k}=1}^{\mathbf{n}}\left[\left\langle\mathrm{e}_{\mathbf{k}}, \mathbf{x}\right\rangle^{\sim}\right]^{2}+\mathrm{i}\langle\mathrm{h}, \mathbf{x}\rangle \sim\right\} \mathrm{d} \nu(\mathbf{x}) \\
& =(2 \pi)^{\frac{-(n+1)}{2}} \int_{\mathbb{R}^{n+1}} \exp \left\{\left[\frac{1-\lambda}{2}\right] \sum_{k=1}^{n} y_{k}^{2}+i \sum_{k=1}^{n+1} c_{k} y_{k}\right\} \\
& \cdot \exp \left\{-\frac{1}{2} \underset{\mathbf{k}=1}{\mathrm{n}+1} \mathrm{y}_{\mathbf{k}}^{2}\right\} \mathrm{dy}_{1} \cdots \mathrm{dy}_{\mathrm{n}+1}
\end{aligned}
$$




$$
\begin{aligned}
& =(2 \pi)^{\frac{-(\mathrm{n}+1)}{2}}\left[\prod_{\mathbf{k}=1}^{\mathrm{n}} \int_{\mathbb{R}} \exp \left\{-\frac{\lambda}{2} \mathrm{y}_{\mathbf{k}}^{2}+i \mathrm{c}_{\mathbf{k}} \mathrm{y}_{\mathbf{k}}\right\} \mathrm{dy_{k }}\right] \\
& \cdot \int_{\mathbb{R}} \exp \left\{-\frac{1}{2} \mathrm{y}_{\mathrm{n}+1}^{2}+\mathrm{ic} \mathrm{n}_{\mathrm{n}+1} \mathrm{y}_{\mathrm{n}+1}\right\} \mathrm{d} \mathrm{y}_{\mathrm{n}+1} \\
& =\lambda^{-\frac{\mathrm{n}}{2}} \exp \left\{-\sum_{\mathbf{k}=1}^{\mathrm{n}} \frac{\mathrm{c}_{\mathbf{k}}^{2}}{2 \lambda}-\frac{\mathrm{c}_{\mathrm{n}+1}^{2}}{2}\right\} \\
& =\lambda^{\frac{-\mathrm{n}}{2}} \exp \left\{\left[\frac{\lambda-1}{2 \lambda}\right] \sum_{\mathbf{k}=1}^{\mathrm{n}}\left[\left\langle\mathrm{e}_{\mathbf{k}}, \mathrm{h}\right\rangle\right]^{2}-\frac{\|\mathrm{h}\|^{2}}{2}\right\} .
\end{aligned}
$$

In the following general theorem, for $F \in \mathscr{F}(B)$, we express the analytic Feynman integral of $\mathrm{F}$ over $\mathrm{B}$ as the limit of a sequence of Wiener integrals on abstract Wiener space.

THEOREM 2. Let $\left\{e_{j}\right\}_{j=1}^{\infty}$ be a CON set of functions in $H$, let $F \in \mathscr{F}(B)$, and let $\left\{\lambda_{j}\right\}_{j=1}^{\infty}$ be a sequence of complex numbers from $C^{+}$such that $\lambda_{j} \rightarrow-$ iq. Then

$$
\mathrm{I}_{\mathrm{a}}^{\mathrm{q}}(\mathrm{F})=\lim _{\mathrm{n} \rightarrow \infty} \lambda_{\mathrm{n}}^{\frac{\mathrm{n}}{2}} \int_{B} \exp \left\{\left[\frac{1-\lambda}{2}\right] \sum_{\mathbf{k}=1}^{\mathrm{n}}\left[\left(\mathrm{e}_{\mathbf{k}}, \mathbf{x}\right)^{\sim}\right]^{2}\right\} \mathrm{F}(\mathbf{x}) \mathrm{d} \nu(\mathbf{x}) .
$$

PROOF. Since $\mathrm{F}$ is in $\mathscr{F}(\mathrm{B})$ we have that

$$
\mathbf{F}(\mathbf{x})=\int_{\mathbf{H}} \exp \left\{\mathrm{i}(\mathbf{h}, \mathbf{x})^{\sim}\right\} \mathrm{d} \mu(\mathrm{h})
$$

for some $\mu \in \mathrm{M}(\mathrm{H})$. By the Fubini theorem and Lemma 1 we obtain that

$$
\begin{aligned}
& \int_{B} \exp \left\{\left[\frac{1-\lambda}{2}\right] \underset{\mathbf{k}=1}{\mathbf{n}}\left[\left(\mathrm{e}_{\mathbf{k}}, \mathbf{x}\right)^{\tilde{2}}\right]^{2}\right\} \mathrm{F}(\mathbf{x}) \mathrm{d} \nu(\mathbf{x}) \\
& =\int_{H} \int_{B} \exp \left\{\left[\frac{1-\lambda}{2}\right] \sum_{\mathbf{k}=1}^{\mathrm{n}}\left[\left(\mathrm{e}_{\mathbf{k}}, \mathbf{x}\right)^{\sim}\right]^{2}+\mathrm{i}(\mathbf{h}, \mathbf{x})^{\sim}\right\} \mathrm{d} \nu(\mathrm{x}) \mathrm{d} \mu(\mathbf{h}) \\
& =\lambda_{\mathbf{n}}^{-\frac{n}{2}} \int_{\mathbf{H}} \exp \left\{\left[\frac{\lambda_{\mathrm{n}}-1}{2 \lambda_{\mathrm{n}}}\right] \underset{\mathbf{k}=1}{\mathrm{n}}\left[\left\langle\mathrm{e}_{\mathrm{k}}, \mathrm{h}\right\rangle\right]^{2}-\frac{\|\mathrm{h}\|^{2}}{2}\right\} \mathrm{d} \mu(\mathrm{h})
\end{aligned}
$$

Next, using the bounded convergence theorem, equation (2.5) and Parseval's relation, it follows that

$$
\begin{aligned}
& \lim _{\mathbf{n} \rightarrow \infty} \lambda_{\mathbf{n}}^{\frac{\mathrm{n}}{2}} \int_{\mathbf{B}} \exp \left\{\left[\frac{1-\lambda}{2}\right] \underset{\mathbf{k}=1}{\sum_{\mathbf{n}}^{\mathrm{n}}}\left[\left(\mathrm{e}_{\mathbf{k}}, \mathbf{x}\right)^{\sim}\right]^{2}\right\} \mathrm{F}(\mathbf{x}) \mathrm{d} \nu(\mathbf{x}) \\
& =\int_{\mathbf{H}} \exp \left\{-\frac{\mathrm{i}}{2 \mathrm{q}}\|\mathrm{h}\|^{2}\right\} \mathrm{d} \mu(\mathrm{h}) \\
& =\mathrm{I}_{\mathrm{a}}^{\mathrm{q}}(\mathrm{F})
\end{aligned}
$$

which concludes the proof of Theorem 2.

A careful examination of the proof of Theorem 2 and using equation (2.6) instead of (2.5) establishes our next theorem.

THEOREM 3. Let $\left\{e_{j}\right\}_{j=1}^{\infty}$ be a CON set of functions in $H$, let $F \in \mathscr{F}(B)$ and let $\lambda \in C^{+}$. Let $\left\{\lambda_{j}\right\}_{j=1}^{\infty}$ be a sequence of complex numbers from $C^{+}$such that $\lambda_{j} \rightarrow \lambda$. Then 


$$
I_{a}^{\lambda}(F)=\lim _{n \rightarrow \infty} \lambda_{n}^{\frac{n}{2}} \int_{B} \exp \left\{\left[\frac{1-\lambda}{2}\right] \underset{k=1}{\sum_{k}^{n}}\left[\left(e_{k}, x\right)\right]^{2}\right\} F(x) d \nu(x) .
$$

Our main result, namely a change of scale formula for Wiener integrals on an abstract Wiener space now follows easily from Theorem 3 above.

THEOREM 4. Let $\rho>0$ be given and let $\left\{e_{j}\right\}_{j=1}^{\infty}$ be a CON set of functions from H. Then for $F \in \mathscr{I}(\mathbf{B})$,

$$
\int_{B} F(\rho x) d \nu(x)=\lim _{n \rightarrow \infty} \rho^{-n} \int_{B} \exp \left\{\left[\frac{\rho^{2}-1}{2 \rho^{2}}\right] \sum_{k=1}^{n}\left[\left(e_{k}, x\right) \tilde{~}\right]^{2}\right\} F(x) d \nu(x) .
$$

PROOF. In Theorem 3 let $\lambda=\rho^{-2}$ and choose $\lambda_{n}=\lambda$ for $n=1,2, \cdots$. Also note that for $\lambda>0$,

$$
\mathrm{I}_{\mathrm{a}}^{\lambda}(\mathrm{F})=\int_{\mathbf{B}} \mathrm{F}\left(\lambda^{-\frac{1}{2}} \mathbf{x}\right) \mathrm{d} \nu(\mathbf{x})
$$

by Definition 1 .

The Banach algebra $\mathscr{F}(B)$ is not closed with respect to pointwise or even uniform convergence $[13, \mathrm{p} .2]$, and thus its closure $\bar{\Im}(\mathrm{B})^{\mu}$ with respect to uniform convergence s-a.e. is a larger space than $\mathscr{F}(\mathrm{B})$. We end this section by showing that equation (3.4) also holds for $\mathrm{F} \in \overline{\mathscr{S}(\mathrm{B})}^{\mu}$.

THEOREM 5. Let $\rho>0$ be given and let $\left\{e_{j}\right\}_{j=1}^{\infty}$ be a CON set of functions in $H$. Then equation (3.4) holds for each $F \in \overline{\mathscr{S}(B)^{\mu}}$.

PROOF. Since $F \in \widetilde{F}^{(B)^{\mu}}$, there exists a sequence $\left\{F_{m}\right\}_{m=1}^{\infty}$ from $\mathscr{F}(B)$ such that $F(x)=\lim _{m \rightarrow \infty} F_{m}(x)$ uniformly s-a.e. on $B$. Also since each $F_{m} \in \mathscr{F}(B), F_{m}(x)$ exists and is bounded s-a.e. on $\mathrm{B}$ for each $\mathrm{m}$. Let $\rho>0$ be given. Frow the definition of uniform convergence s-a.e., it follows that

$$
F(\rho x)=\lim _{m \rightarrow \infty} F_{m}(\rho x) \text { uniformly a.e. on } B
$$

and

$$
\int_{B} F(\rho x) d \nu(x)=\lim _{\mathbf{m} \rightarrow \infty} \int_{B} F_{m}(\rho x) \mathrm{d} \nu(x) .
$$

Now from Lemma 1 with $\lambda=\rho^{-2}$ and $\mathrm{h} \equiv 0$, we obtain that

$$
\int_{B} \exp \left\{\left[\frac{\rho^{2}-1}{2 \rho^{2}}\right] \sum_{\mathbf{k}=1}^{\mathrm{n}}\left[\left(\mathrm{e}_{\mathbf{k}}, \mathbf{x}\right)^{\sim}\right]^{2}\right\} \mathrm{d} \nu(\mathbf{x})=\rho^{\mathrm{n}} .
$$

By (3.5), there exists $M>0$ and an invariant null subset $E$ of $B$ (i.e., $\nu(\rho E)=0$ for all $\rho>0$ ) such that for all $\mathrm{m}$ and all $\mathrm{x} \in \mathrm{B}-\mathrm{E}$

$$
\left|F_{m}(x)\right| \leq M \text { and }|F(x)| \leq M
$$

Hence, using (3.7) and (3.8) we obtain

$$
\begin{aligned}
& \mid \rho^{-\mathrm{n}} \int_{\mathrm{B}} \exp \left\{\left[\frac{\rho^{2}-1}{2 \rho^{2}}\right] \sum_{\mathbf{k}=1}^{\mathrm{n}}\left[\left(\mathrm{e}_{\mathbf{k}}, \mathbf{x}\right)^{\sim}\right]^{2}\right\} \mathrm{F}_{\mathbf{m}}(\mathbf{x}) \mathrm{d} \nu(\mathbf{x}) \\
& -\rho^{-\mathbf{n}} \int_{\mathrm{B}} \exp \left\{\left[\frac{\rho^{2}-1}{2 \rho^{2}}\right] \sum_{\mathbf{k}=1}^{\mathbf{n}}\left[\left(\mathrm{e}_{\mathbf{k}}, \mathbf{x}\right)^{\tilde{2}}\right]^{2}\right\} \mathrm{F}(\mathbf{x}) \mathrm{d} \nu(\mathbf{x}) \mid
\end{aligned}
$$




$$
\begin{aligned}
& \leq \rho^{-\mathrm{n}} \int_{\mathbf{B}} \exp \left\{\left[\frac{e^{2}-1}{2 \rho^{2}}\right] \underset{\mathbf{k}=1}{\mathrm{n}}\left[\left(\mathrm{e}_{\mathbf{k}}, \mathrm{x}\right)^{\sim}\right]^{2}\right\}\left|\mathrm{F}_{\mathrm{m}}(\mathrm{x})-\mathrm{F}(\mathrm{x})\right| \mathrm{d} \nu(\mathrm{x}) \\
& \leq 2 \mathrm{M} .
\end{aligned}
$$

Finally, using Theorem 2, the iterated limits theorem and the dominated convergence theorem, we obtain

$$
\begin{aligned}
& \int_{\mathbf{B}} \mathrm{F}(\rho \mathrm{x}) \mathrm{d} \nu(\mathbf{x})=\lim _{\mathbf{m} \rightarrow \infty} \int_{\mathbf{B}} \mathrm{F}_{\mathrm{m}}(\rho \mathbf{x}) \mathrm{d} \nu(\mathbf{x}) \\
& =\lim _{\mathrm{m} \rightarrow \infty} \lim _{\mathrm{n} \rightarrow \infty} \rho^{-\mathrm{n}} \int_{\mathrm{B}} \exp \left\{\left[\frac{e^{2}-1}{2 \rho^{2}}\right] \underset{\mathbf{k}=1}{\mathrm{n}}\left[\left(\mathrm{e}_{\mathbf{k}}, \mathbf{x}\right)^{\sim}\right]^{2}\right\} \quad \mathrm{F}_{\mathrm{m}}(\mathrm{x}) \mathrm{d} \nu(\mathrm{x}) \\
& =\lim _{n \rightarrow \infty} \lim _{m \rightarrow \infty} \rho^{-n} \int_{B} \exp \left\{\left[\frac{e^{2}-1}{2 \rho^{2}}\right] \underset{k=1}{\sum}\left[\left(e_{k}, x\right)\right]^{2}\right\} \quad F_{m}(x) d \nu(x) \\
& =\lim _{\mathbf{n} \rightarrow \infty} \rho^{-\mathrm{n}} \int_{\mathrm{B}} \exp \left\{\left[\frac{e^{2}-1}{2 \rho^{2}}\right] \sum_{\mathbf{k}=1}^{\mathrm{n}}\left[\left(\mathrm{e}_{\mathbf{k}}, \mathbf{x}\right)^{\tilde{2}}\right]^{2}\right\} \mathrm{F}(\mathbf{x}) \mathrm{d} \nu(\mathbf{x})
\end{aligned}
$$

and so equation (3.4) holds for each $\mathrm{F} \in \overline{\mathscr{F}(\mathrm{B})}^{\mu}$.

\section{COROLLARIES.}

In this section we give various corollaries which show that Theorem 4 above, giving the change of scale formula (3.4), is indeed a very general theorem since it holds for all functions $\mathrm{F}$ in $\mathscr{F}(\mathrm{B})$ where $(\mathrm{H}, \mathrm{B}, \nu)$ is any abstract Wiener space. Below we list results of three types.

a. Classical Wiener Spaces. Fix $\mathrm{T}>0$ and let $\mathrm{H}_{\mathrm{O}}=\mathrm{H}_{\mathrm{o}}[0, \mathrm{~T}]$ be the space of $\mathbb{R}$-valued functions $\gamma$ on $[0, T]$ which are absolutely continuous and whose derivative $\mathrm{D} \gamma$ is in $\mathrm{L}_{2}[0, \mathrm{~T}]$. The inner product on $\mathrm{H}_{\mathrm{o}}$ is given by

$$
\langle\gamma, \beta\rangle_{\mathrm{H}_{\mathrm{o}}}=\int_{0}^{\mathrm{T}}(\mathrm{D} \gamma)(\mathrm{s})(\mathrm{D} \beta)(\mathrm{s}) \mathrm{ds} .
$$

$\mathrm{H}_{\mathrm{o}}$ is a separable Hilbert space over $\mathbb{R}$. Let $\mathrm{B}_{\mathrm{o}}=\mathrm{B}_{0}[0, T]$ be the space $\mathrm{C}_{0}[0, T]$ of all continuous functions $x$ on $[0, T]$ with $x(0)=0$ and equip $B_{0}$ with the sup norm. Let $\nu_{0}$ be classical Wiener measure. Note that if $\left\{e_{j}\right\}_{j=1}^{\infty}$ is a CON set in $H_{0}$ then $\left\{D_{j}\right\}_{j=1}^{\infty}$ is a CON set in $L_{2}[0, T]$ and $\left(e_{j}, x\right)^{\sim}$ equals the Paley-Wiener-Zygmund stochastic integral $\mathrm{T}$ $\int_{0}\left(D e_{j}\right)(s) \tilde{d} x(s)$ for s-a.e. $x \in B_{\circ}$.

Let $\mathrm{m}$ be a positive integer. Let $\mathrm{H}=\mathrm{H}_{0}^{\mathrm{m}}=\mathrm{H}_{0}^{\mathrm{m}}[0, \mathrm{~T}]$ with inner product

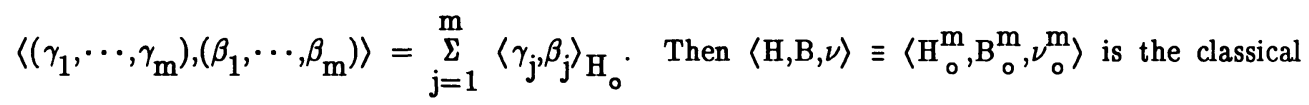
Wiener space in $\mathrm{m}$ dimensions and $\left(\mathrm{B}_{\circ}^{\mathrm{m}}, \nu_{0}^{\mathrm{m}}\right)$ is the classical Wiener space of paths in $\mathbb{R}^{\mathrm{m}}$. Let $S=S(m)$ be the space of functions on $C_{0}^{m}[0, T]=B_{0}^{m}$ of the form

$$
F(\vec{x})=\int_{L_{2}^{m}[0, T]} \exp \left\{i \sum_{j=1}^{m} \int_{0}^{\mathrm{m}} \mathrm{v}_{j}(s) \tilde{d} x_{j}(s)\right\} \mathrm{d} \mu(\vec{v})
$$

for s-a.e. $\vec{x}=\left(x_{1}, \cdots, x_{m}\right) \in B_{o}^{m}$ for some $\mu \in M\left(L_{2}^{m}[0, T]\right)$. 
Our first corollary contains Theorem 2 of [7] while our second corollary contains Theorem 4 of [7].

COROLLARY 1. Let $\left\{\phi_{j}\right\}_{j=1}^{\infty}$ be a CON set of functions on $[0, T]$. Then for each $\rho>0$ and each $\mathrm{F} \in \mathrm{S}(\mathrm{m})$

$$
\int_{B_{0}^{m}} F(\rho \vec{x}) \mathrm{d} \nu(\overrightarrow{\mathbf{x}})=\lim _{\mathbf{n} \rightarrow \infty} \rho^{-\mathrm{mn}} \int_{\mathbf{B}_{0}^{\mathbf{m}}} \exp \left\{\frac{\rho^{2}-1}{2 \rho^{2}} \sum_{\mathbf{j}=1}^{\mathrm{m}} \sum_{\mathbf{k}=1}^{\mathrm{n}}\left[\int_{0}^{\mathrm{T}} \phi_{\mathbf{k}}(\mathrm{s}) \tilde{\mathrm{d}} \mathrm{x}_{\mathrm{j}}(\mathrm{s})\right]^{2}\right\} \mathrm{F}(\overrightarrow{\mathbf{x}}) \mathrm{d} \nu(\overrightarrow{\mathbf{x}}) .
$$

PROOF. Let $e_{k}(t)=\int_{0}^{t} \phi_{k}(s) d s$ on $[0, T]$ and use Theorem 4 and the fact that $S(m)$ and $\mathscr{F}\left(\mathrm{B}_{0}^{\mathrm{m}}\right)$ are isometrically isomorphic $[14,21]$.

COROLLARY 2. Equation (4.1) holds for all $\mathrm{F} \in \overline{\mathrm{S}(\mathrm{m})}^{\mu}$.

b. $\quad \mathrm{N}$-parameter Wiener Space. Let $\mathrm{B}_{\mathrm{N}, 0}=\mathrm{C}_{\mathrm{o}}\left([0, \mathrm{~T}]^{\mathrm{N}}\right)$ be the space of all $\mathbb{R}$-valued continuous functions $x$ on $[0, T]^{N}$ such that $x\left(t_{1}, \cdots, t_{N}\right)=0$ whenever at least one of $\mathrm{t}_{1}, \cdots, \mathrm{t}_{\mathrm{N}}$ equals zero. Let $\nu_{\mathrm{N}, 0}$ denote $\mathrm{N}$-parameter Wiener measure on $\left(\mathrm{B}_{\mathrm{N}, 0}, \mathcal{B}\left(\mathrm{B}_{\mathrm{N}, 0}\right)\right)$. The 2-parameter Wiener space $\left(\mathrm{C}_{0}\left([0, T]^{2}\right), \mathscr{B}\left(\mathrm{C}_{0}\left([0, \mathrm{~T}]^{2}\right)\right), \nu_{2,0}\right)$ is often called Yeh-Wiener space, and the sample functions $x$ in $\mathrm{C}_{0}\left([0, T]^{2}\right)$ are often called Brownian surfaces or Brownian sheets.

Let $\mathrm{H}_{\mathrm{N}, 0}$ denote the set of all functions $\gamma:[0, T]^{N} \rightarrow \mathbb{R}$ for which there exists $\mathrm{g}$ in $\mathrm{I}_{2}\left([0, T]^{\mathrm{N}}\right)$ such that for all $\left(\mathrm{s}_{1}, \cdots, \mathrm{s}_{N}\right) \in[0, T]^{N}$,

$$
\gamma\left(t_{1}, \cdots, t_{N}\right)=\int_{0}^{t_{1}} \cdots \int_{0}^{t_{N}} g\left(s_{1}, \cdots, s_{N}\right) d s_{N} \cdots d s_{1} .
$$

The inner product on $\mathrm{H}_{\mathrm{N}, 0}$ is defined by

$$
\langle\gamma, \beta\rangle_{\mathrm{H}_{\mathrm{N}, 0}}=\int_{0}^{\mathrm{T}} \cdots \int_{0}^{\mathrm{T}}\left[\frac{\partial^{\mathrm{N}} \gamma}{\partial \mathrm{s}_{1} \cdot \partial_{\mathrm{N}} \mathrm{N}}\right]\left[\frac{\partial^{\mathrm{N}} \beta}{\partial \mathrm{s}_{1} \cdots \partial \mathrm{s}_{\mathrm{N}}}\right] \mathrm{ds}_{1} \cdots \mathrm{ds} \mathrm{s}_{\mathrm{N}}
$$

and $\left(\mathrm{H}_{\mathrm{N}, 0}, \mathrm{~B}_{\mathrm{N}, 0}, \nu_{\mathrm{N}, 0}\right)$ is an abstract Wiener space. Again

$$
(\gamma, \mathrm{x})^{\sim}=\int_{0}^{\mathrm{T}} \cdots \int_{0}^{\mathrm{T}} \mathrm{g}\left(\mathrm{s}_{1}, \cdots, \mathrm{s}_{\mathrm{N}}\right) \tilde{\mathrm{d}} \mathrm{x}\left(\mathrm{s}_{1}, \cdots, \mathrm{s}_{\mathrm{N}}\right)
$$

for s-a.e. $x \in B_{N, 0}$.

Let $S_{N}(1)$ be the space of functions $F$ on $B_{N, 0}$ of the form

$$
\mathrm{F}(\mathrm{x})=\int_{\mathrm{L}_{2}\left([0, \mathrm{~T}]^{\mathrm{N}}\right)} \exp \left\{\mathrm{i} \int_{0}^{\mathrm{T}} \cdots \int_{0}^{\mathrm{T}} \mathrm{v}\left(\mathrm{s}_{1}, \cdots, \mathrm{s}_{\mathrm{N}}\right) \tilde{\mathrm{d} x}\left(\mathrm{~s}_{1}, \cdots, \mathrm{s}_{\mathrm{N}}\right)\right\} \mathrm{d} \mu(\mathrm{v})
$$

for s-a.e. $x$ in $B_{N, 0}$ for some $\mu \in M\left(L_{2}[0, T]^{N}\right)$.

COROLLARY 3. Let $\left\{\phi_{j}\right\}_{j=1}^{\infty}$ be a CON set of functions on $[0, T]^{N}$. Then for each $\rho>0$ and each $F \in S_{N}(1)$, 


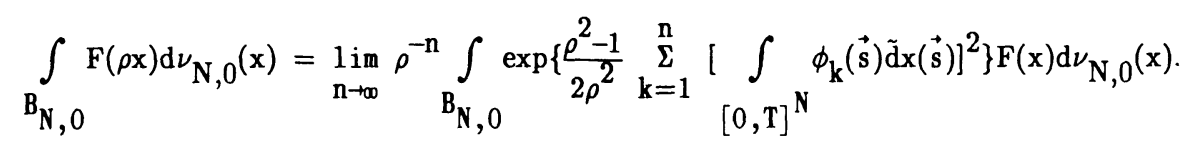

PROOF. Simply let $e_{k}\left(t_{1}, \cdots, t_{N}\right)=\int_{0}^{t_{1}} \cdots \int_{0}^{t_{N}} \phi_{k}\left(s_{1}, \cdots, s_{N}\right) d s_{N} \cdots d_{1}$ and use Theorem 4 and the fact that $\mathrm{S}_{\mathrm{N}}(1)$ and $\mathscr{F}\left(\mathrm{B}_{\mathrm{N}, 0}\right)$ are isometrically isomorphic.

COROLLARY 4. (Theorem 4.2 of $[8]$ ). This is the case $\mathrm{N}=2$ in Corollary 3 above.

In our next corollary we consider $m$ copies of $N$-parameter Wiener space $B_{N, 0}$. Yoo and Chang in [18] worked with functions in $\mathrm{S}_{\mathrm{N}}(\mathrm{m})$ and the corresponding Banach algebra $\mathscr{F}\left(\mathrm{B}_{\mathrm{N}, 0}^{\mathrm{m}}\right)$.

COROLLARY 5. Let $(\mathrm{H}, \mathrm{B}, \nu)=\left(\mathrm{H}_{\mathrm{N}, 0}^{\mathrm{m}}, \mathrm{B}_{\mathrm{N}, 0}^{\mathrm{m}}, \nu_{\mathrm{N}, 0}^{\mathrm{m}}\right)$. Let $\left\{\phi_{\mathrm{j}}\right\}_{\mathrm{j}=1}^{\mathrm{d}}$ be a a CON set of functions on $[0, T]^{N}$. Then for each $\rho>0$ and each $F$ in $S_{N}(m)$,

$$
\begin{aligned}
& \int \mathrm{F}(\rho \overrightarrow{\mathbf{x}}) \mathrm{d} \nu_{\mathrm{N}, 0}^{\mathrm{m}}(\overrightarrow{\mathbf{x}}) \\
& \mathrm{B}_{\mathrm{N}, 0}^{\mathrm{m}} \\
& =\lim _{\mathbf{n} \rightarrow \infty} \rho^{-\mathrm{mn}} \int_{\mathbf{B}_{\mathrm{N}, 0}^{\mathrm{m}}} \exp \left\{\frac{\rho^{2}-1}{2 \rho^{2}} \sum_{\mathrm{j}=1}^{\mathrm{m}} \sum_{\mathbf{k}=1}^{\mathrm{n}}\left[\int_{[0, \mathrm{~T}]^{N}} \phi_{\mathbf{k}}(\overrightarrow{\mathrm{s}}) \tilde{\mathrm{d}} \mathrm{x}_{\mathrm{j}}(\overrightarrow{\mathrm{s}})\right]^{2}\right\} \mathrm{F}(\overrightarrow{\mathrm{x}}) \mathrm{d} \nu_{\mathrm{N}, 0}^{\mathrm{m}}(\overrightarrow{\mathrm{x}}) .
\end{aligned}
$$

c. Other Spaces. Ahn et al [17] established a very general theorem insuring that many functions of interest in Feynman integration theory and quantum mechanics are in $\mathscr{F}(\mathrm{B})$ for various abstract Wiener spaces $(\mathrm{H}, \mathrm{B}, \nu)$.

COROLLARY 6. All of the functions discussed in Corollaries 1-11 of [17] satisfy the change of scale formula (3.4) where $\left\{\mathrm{e}_{\mathrm{j}}\right\}_{\mathrm{j}=1}^{\infty}$ is a CON set of functions in the corresponding Hilbert space $\mathrm{H}$.

COROLLARY 7. All of the functions considered in [6,9-16] satisfy the change of scale formula (3.4) where $\left\{e_{j}\right\}_{j=1}^{\infty}$ is a CON set of functions in the appropriate Hilbert space $\mathrm{H}$.

PROOF. These functions all belong to some $\mathscr{F}(B)$ or $S(m)$.

ACKNOWLEDGEMENT. Research of the first author was supported in part by the Korea Science and Engineering Foundation and the Ministry of Education.

\section{REFERENCES}

1. CAMERON, R.H. and MARTIN, W.T. The Behavior of Measure and Measurability Under Change of Scale in Wiener Space, Bull. Amer. Math. Soc. $\underline{53}(1947), 130-137$.

2. CAMERON, R.H. The Translation Pathology of Wiener Space, Duke Math. J. 21(1954), 623-628.

3. JOHNSON, G.W. and SKOUG, D.L. Scale-Invariant Measurability in Wiener Space, Pacific J. Math. $\underline{83}(1979), 157-176$. 
4. CHANG, K.S. Scale-Invariant Measurability in Yeh-Wiener Space, J. Korean Math. Soc. 19(1982), 61-67.

5. CHUNG, D.M. Scale-Invariant Measurability in Abstract Wiener Space, Pacific J. Math. $130(1987), 27-40$.

6. CAMERON, R.H. and STORVICK, D.A. Relationships Between the Wiener Integral and the Analytic Feynman Integral, Supplemento ai Rendiconti del Circolo Matematico di Palermo, Serie II - numero $17(1987), 117-133$.

7. CAMERON, R.H. and STORVICK, D.A. Change of Scale Formulas for Wiener Integral, Supplemento ai Rendiconti del Circolo Matematico di Palermo, Serie II numero 17 (1987), 105-115.

8. YOO, I. Sequential Yeh-Feynman Integrals, Doctoral Thesis, Yonsei University, Seoul, Korea (1987).

9. CHANG, K.S., JOHNSON, G.W. and SKOUG, D.L. Necessary and Sufficient Conditions for the Fresnel Integrability of Certain Classes of Functions, $\underline{\text { J. Korean }}$ Math. Soc. 21(1984), 21-29.

10. CHANG, K.S., JOHNSON, G.W. and SKOUG, D.L. Functions in the Fresnel Class, Proc. Amer. Math. Soc. 100(1987), 309-318.

11. CHANG, K.S., JOHNSON, G.W. and SKOUG, D.L. Necessary and Sufficient Conditions for Membership in the Banach Algebra $S$ for Certain Classes of Functions, Supplemento ai Rendiconti del Circolo Matematico di Palermo, Serie II-numero 17(1987), 153-171.

12. CHANG, K.S., JOHNSON, G.W. and SKOUG, D.L. Functions in the Banach Algebra S $(\nu)$, J. Korean Math. Soc. 24 (1987), 151-158.

13. JOHNSON, G.W. and SKOUG, D.L. Stability Theorems for the Feynman Integral, Supplemento ai Rendiconti del circolo Matematico di Palermo, Serie II-numero $\underline{8}(1985), 361-367$.

14. KALLIANPUR, G. and BROMLEY, C. Generalized Feynman Integrals Using Analytic Continuation in Several Complex Variables, In Stochastic Analysis and Applications (Ed. M.H. Pinsky) 433-450, Dekker, New York, 1984.

15. KALlianPUR, G., KANNAN, D. and KARANDIKAR, R.L., Analytic and Sequential Feynman Integrals on Abstract Wiener and Hilbert Spaces and a Cameron-Martin Formula, Ann. Inst. Henri Poincaré 21 (1985), 323-361.

16. CAMERON, R.H. and STORVICK, D.A. Some Banach Algebras of Analytic Feynman Integrable Functionals, In Analytic Functions Kozubnik 1979, Lecture Notes in Math. Vol. 798, Springer-Verlag, Berlin, 1980, 18-67.

17. AHN, J.M., JOHNSON, G.W. and SKOUG, D.L. Functions in the Fresnel Class of an Abstract Wiener Space, J. Korean Math. Soc. 28 (1991), 245-265.

18. YOO, I and CHANG, K.S. Notes on Analytic Feynman Integrable Functions, to appear in Rocky Mountain J. of Math.

19. GROSS, L. Abstract Wiener Space, Proc. 5th Berkeley Sympos. Math. Stat. Prob. $\underline{2}(1965), 31-42$.

20. KUO, H.H. Gaussian Measures in Banach Spaces, Lecture Notes in Math. Vol. 463, Springer-Verlag, Berlin, 1975.

21. JOHNSON, G.W. The Equivalence of Two Approaches to the Feynman Integral, J. Math. Phys. 23(1982), 2090-2096. 


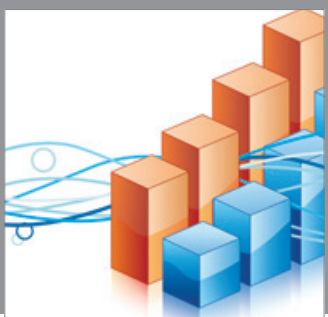

Advances in

Operations Research

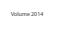

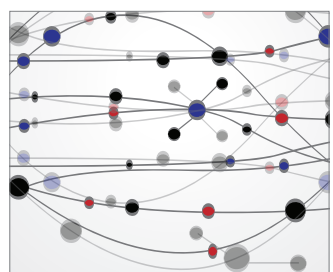

\section{The Scientific} World Journal
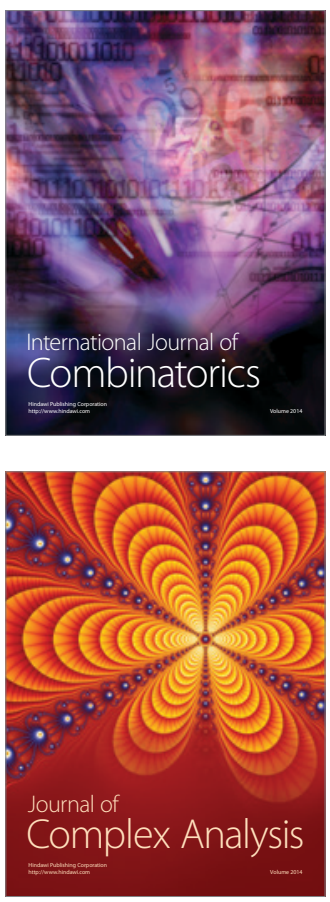

International Journal of

Mathematics and

Mathematical

Sciences
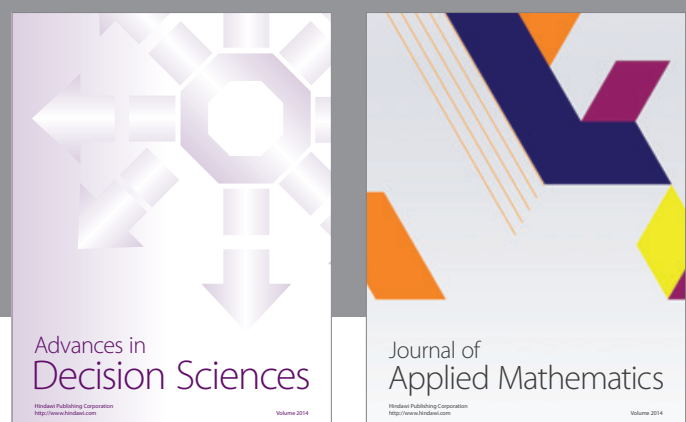

Journal of

Applied Mathematics
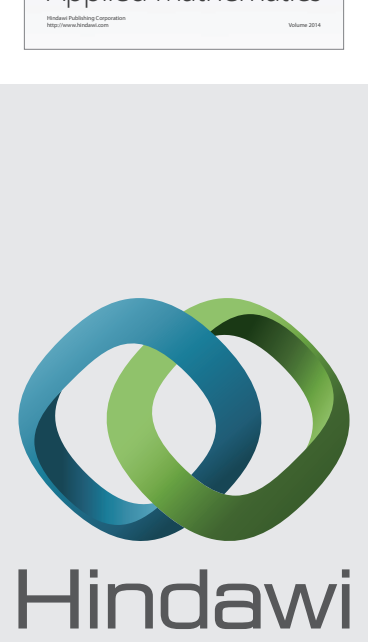

Submit your manuscripts at http://www.hindawi.com
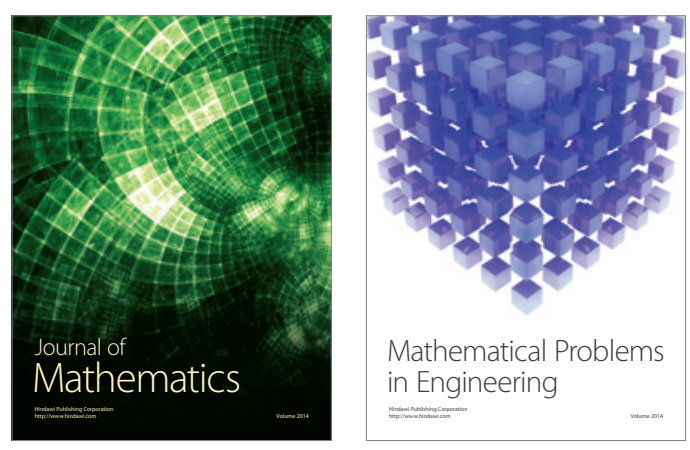

Mathematical Problems in Engineering
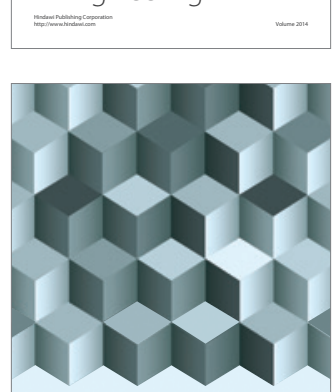

Journal of

Function Spaces
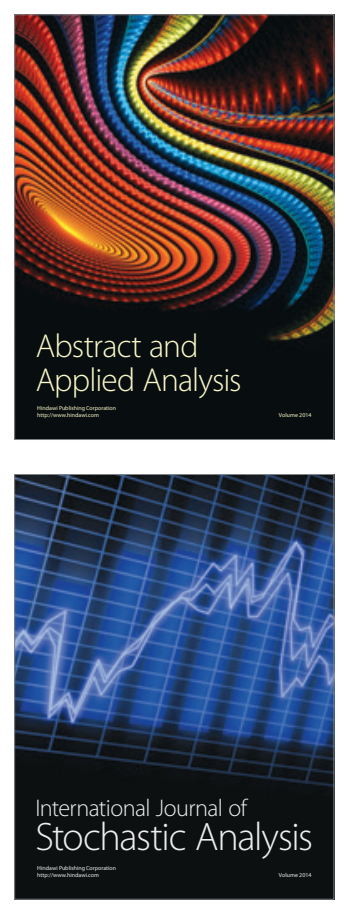

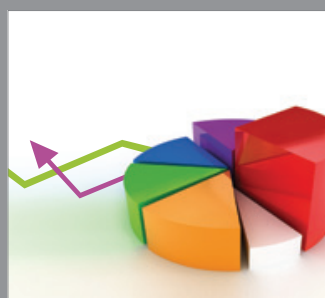

ournal of

Probability and Statistics

Promensencen
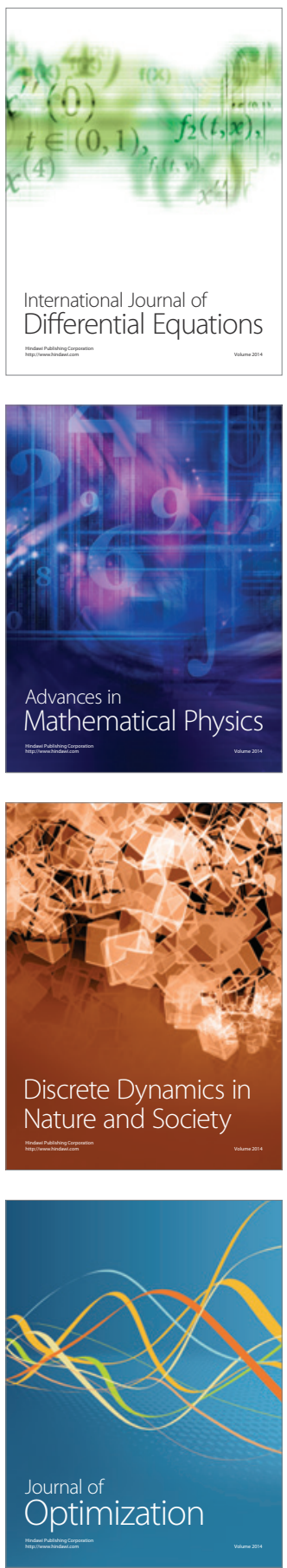